\title{
Restrained fall velocity of suspended solids particles sedimentation in still water
}

\author{
Irina Markova ${ }^{1,{ }^{*}}$ and Yuliya Bryanskaya ${ }^{1}$ \\ ${ }^{1}$ Moscow State University of Civil Engineering (MGSU) 129337, 26, Yaroslavskoye Shosse, \\ Moscow, Russia
}

\begin{abstract}
The deposition rate is sufficiently studied in the case of single particles or in conditions of low concentrations of suspended matter, in which the precipitating particles do not affect each other. In the article, the method of calculation of the rate of the constrained deposition is offered, comparative calculations of the rate of the free and constrained deposition of particles are executed, the settlement results reflecting essential difference of the speed of the constrained and free deposition of particles are presented. The factors influencing the rate of confined deposition, among which the main one is the concentration of suspended matter in water, are considered. The experimental data confirming the reduction of speed of the constrained deposition of more than 3 times in a five-fold increase in the concentration of suspended solids is given. The dependence of the strained deposition rate of particles on the concentration of the suspension is obtained.
\end{abstract}

\section{Introduction}

The ecological state of water bodies largely depends on the effluent entering them, including from surface runoff. The process of sedimentation of sediment is an important part of the formation of channel sediments. As a rule, it is carried out in low-flow periods, when flow rates decrease, and the flow cannot transport suspended matter coming from urban areas. Currently, issues related to the deposition of single particles of the correct form are theoretically and experimentally well studied. The flow around and sedimentation of particles of complex and geometrically irregular shape is a significant difficulty in theoretical description and, as a rule, is studied experimentally.

\section{Methods and Materials}

Let us consider in more detail the process of "constrained" sedimentation of suspensions. If the volume concentration of particles $\mathrm{C}$, then, falling into the lower layers, they "displace" upward the corresponding liquid volume, as a result of which upward "compensatory" movement of the liquid occurs. If we assume that the compensation fluid flow is through the entire cross section $\omega_{0}$, then the volume of the solid phase flowing down per unit time, will be

\footnotetext{
* Corresponding author: markova@mgsu.ru
} 


$$
W_{T}=C W \omega_{0}
$$

An equal volume of fluid will be forced upwards, creating an upward "compensatory" flow at a rate

$$
V_{K}=\frac{W_{T}}{\omega_{0}}=C W
$$

At the same time, the subsequent layers of particles will be deposited in the ascending flow and in the fixed coordinate system, according to M.A.Velikanov [8] will have speed

$$
W_{C}=W-V_{K}=W(1-C)
$$

where $V \kappa$ - the speed of "compensatory" movement, indicating a decrease in the deposition rate with increasing concentration of suspended matter $C$.

The deposition rate decreases due to the fact that the layer of particles moving after the first layer (front) will experience the effect of an upward compensating fluid flow. However, the experiments of B.M. Levine [8] found a more complex relationship between the rate of constrained sedimentation and the concentration of suspended matter, which indicates the need for additional consideration of this issue.

To estimate the speed of constrained sedimentation of soil in still water at low concentrations, B.M. Levin proposed empirical dependence

$$
W_{\text {c.s. }}=W_{0}\left(1-C^{n}\right)
$$

where $\mathrm{n}$ is an empirical coefficient depending on the average particle diameter of the soil.

According to P.V. Lyashchenko [8] exponent $n$ varies from 2.5 to 3.8 and is close on average 3. Researches by B.M. Levin et al. [1-13] showed that for shallow soils, the rate of constrained sedimentation is subject to

$$
W_{c . s .}=W_{0}\left(1-\alpha C^{n}\right)
$$

where the value is $a \approx 2.5, n \approx 0.5$ for particles with a particle size from $20 \mu$ to $100 \mu$.

Considering in more detail the movement of particles following the "first" layer, you can see that some of the grains of the "second" layer will move in the wakes of the grains of the "first" layer, the other part in the "windows" between the grains the first "layer, while the speed of the upward compensatory flow in the" windows "(Fig. 1.) is determined as follows:

$$
V_{K .0}=\frac{W_{T}}{\omega_{O K}}=\frac{W_{T}}{\omega_{0}(1-C)}=\frac{C W_{\omega_{0}}}{\omega_{0}(1-C)}=W \frac{C}{1-C}
$$

where $W_{T}$ - the volume of the solid phase coming down per unit of time; $\omega_{0}$ - sectional area; $\omega_{\mathrm{OK}}$ - sectional area of the "window"; $C$ - concentration.

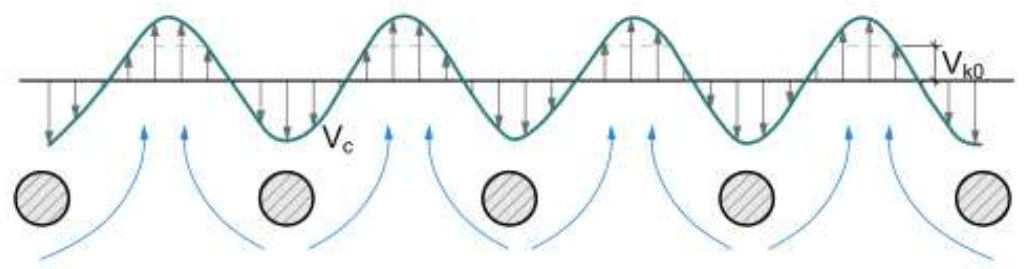

Fig. 1. Scheme for calculating the compensation flow with constrained deposition.

If we assume that the bulk of the particles of the second layer will move in the zones of windows, their speed in a fixed coordinate system will be equal to

$$
W_{C . S}=W-V_{K .0}=W\left(1-\frac{C}{1-C}\right)=W \frac{1-2 C}{1-C}
$$


Comparison of the calculation for this dependence with the calculation by M.A. Velikanov, given in Table.1, shows that the obtained dependence reveals a more intensive decrease in the rate of constrained sedimentation with increasing concentration than the dependence of M. A. Velikanov. A comparison of the calculated data for the rate of constrained deposition is shown in Fig. 3.

Table 1. Comparison of the calculation.

\begin{tabular}{|c|c|c|}
\hline \multirow{2}{*}{$\mathrm{C}$} & \multicolumn{2}{|c|}{$\mathbf{w} \mathbf{c s} / \mathbf{w}$} \\
\cline { 2 - 3 } & Calculation by the formula 7 & Calculation by M.A. Velikanov \\
\hline 0,05 & 0,947 & 0,95 \\
\hline 0,1 & 0,89 & 0,90 \\
\hline 0,2 & 0,75 & 0,80 \\
\hline 0,3 & 0,52 & 0,70 \\
\hline 0,4 & 0,33 & 0,60 \\
\hline
\end{tabular}

However, at this rate only a fraction of particles equal to $(1-C)$ of the total number of particles of the second layer will precipitate. The remaining part of the particles of the second layer will move "in the tracks" behind the particles of the first layer. With a significant concentration of particles, the distance between the particles of the first layer and the "in the tracks" particles of the second layer will be small, and the particles of the "second" layer will move in a fixed coordinate system with speed

$$
W+V_{C}
$$

where $V_{C}$ is the velocity of the return flow in the wake behind the particles of the first row (Fig. 2.).

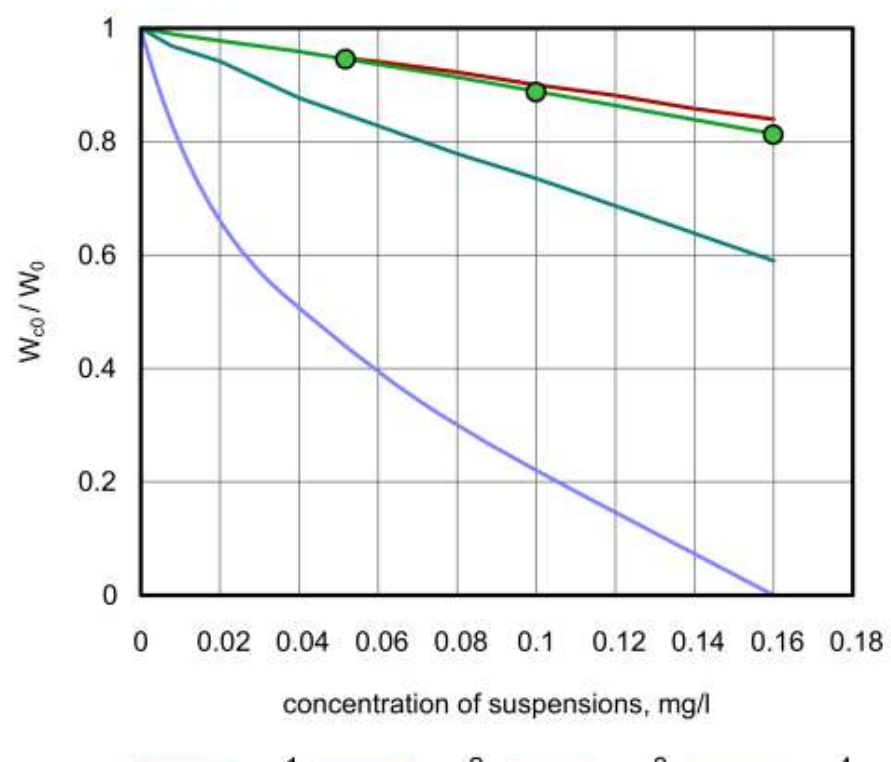

Fig. 2. The speed of the constrained deposition of fine suspension $(d<100 \mathrm{mk}): 1$ - M.A.Velikanov; 2 - Calculation by the formula 5; 3 - P.V. Lyashchenko; 4 - B.M. Levine. 
This circumstance will lead to the fact that the particles of the second layer "catch up" particles of the "first" layer and unite under the action of adhesion forces, forming flakes, or move side by side and constrain the "windows" between the particles of the first row.

Thus, rather quickly, the speed of this fraction of particles will become equal to $W$. At the same time, the area of "windows" will decrease and become equal $\omega_{0}(1-2 C)$, and the speed of the upward compensatory flow will increase to

$$
V_{K .1}=\frac{W_{T}}{\omega_{0}(1-2 C)}=\frac{C W_{\omega_{0}}}{\omega_{0}(1-2 C)}=W \frac{C}{1-2 C}
$$

Then the average deposition rate of the particles of the first and second layer can be defined as

$$
W * 2 C+W\left(1-\frac{C}{1-2 C}\right)(1-2 C)=W\left[2 C+\frac{(1-3 C)(1-2 C)}{1-2 C}\right]=W(1-C)
$$

In fact, the average volume deposition rate of particles turned out to be in this formulation coinciding with the estimate by M.A. Velikanov.

With an increase in the rate of upward compensation flow in the "windows", the particles in these zones will lag behind the particles of the first layer, as a result of which the "cloud" of particles will lengthen, which is confirmed by observations, but is usually associated only with the presence of particles of various sizes in suspension.

It should be noted that the concentration of suspended matter in surface wastewater, reaching 1000-3000 $\mathrm{mg} / \mathrm{l}$ and being rather high from the point of view of sanitary standards, remains as a fraction of the total volume a small value, not exceeding $0.001-0.003$. Thus, the volume of suspended particles in surface wastewater reaches only $0.1-0.3 \%$ of the total volume, therefore, the overwhelming majority of the suspended matter grains of the "second" layer move precisely in the "window" zones. With such a slight constraint by the particles of the total cross section, the process of settling essentially depends on the initial concentration of the suspension (Fig. 3).

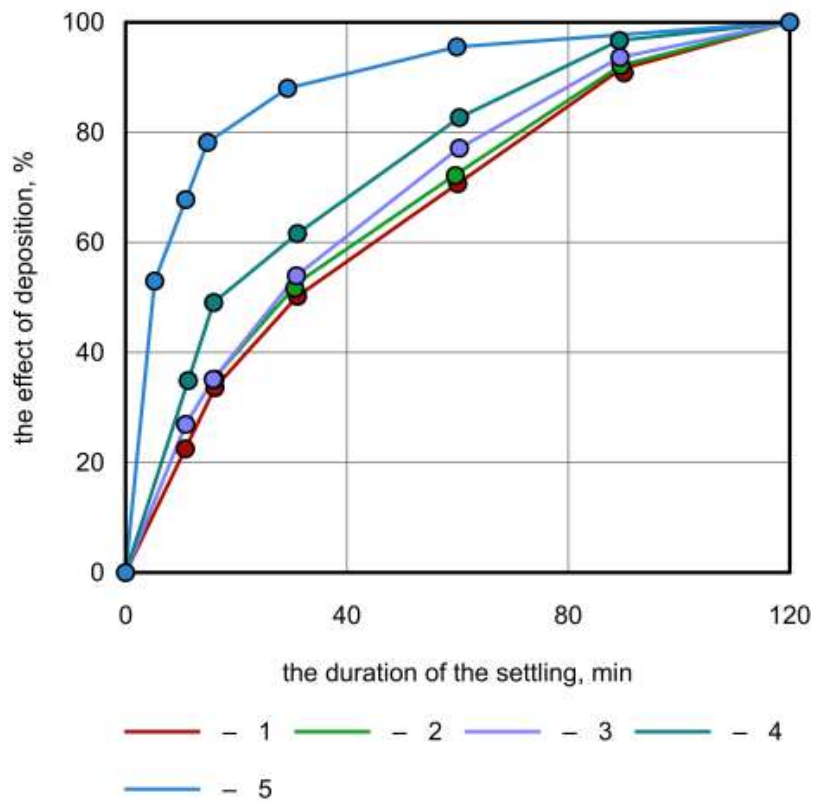

Fig. 3. The deposition curves of suspended solids contained in domestic wastewater (data SV Yakovlev). With an initial concentration of suspended solids: $1-0.5-1.5 \mathrm{ml} / 1 ; 2-1.6-2.5 \mathrm{ml} / \mathrm{l} ; 3$ 2.6-3.4 $\mathrm{ml} / 1 ; 4$ - 3.5-4.7 $\mathrm{ml} / 1 ; 5-5.5-16.3 \mathrm{ml} / 1$. 
However, a more rapid clarification of polluted waters with a high concentration of suspended matter is contradicted as an estimate by M.A. Velikanov, and other experimental data. Calculations show (Table 1) that taking into account the effect of constraint on the deposition rate should be introduced with a significant concentration of suspension $\mathrm{C}>0.10$. This can be observed in the flood, during emergency discharges, and in the hydraulic cleaning of the channels from contaminated bottom soils.

Analytical calculations on the speed of free and constrained sedimentation of individual particles and suspensions were confirmed experimentally by a series of experiments (Table $2,3)$ conducted with model water-soil media of various concentrations containing suspensions of various origin, size, weight and density. The data on the deposition of particles are presented in Table 3, show that there is a "lengthening of the cloud", while particles of the 1 st layer are deposited on average within 4 s., while particles in the "tail of the cloud" are deposited in $15-25 \mathrm{~s}$.

Table 2. Free sedimentation of particles.

\begin{tabular}{|c|c|c|c|c|c|c|}
\hline No & $\mathrm{d}$ & $\mathrm{h}$ & $\mathrm{t}, \mathrm{s}$ & $v, \mathrm{~m} / \mathrm{s}$ & $v^{*} 2, \mathrm{~m} / \mathrm{s}$ & $\operatorname{Re}$ \\
\hline 1 & \multirow[b]{10}{*}{0,00075} & \multirow[b]{10}{*}{0,4} & 4,15 & 0,096 & 0,0093 & 72,29 \\
\hline 2 & & & 4,1 & 0,098 & 0,0095 & 73,17 \\
\hline 3 & & & 3,95 & 0,101 & 0,0103 & 75,97 \\
\hline 4 & & & 4,35 & 0,092 & 0,0085 & 68,97 \\
\hline 5 & & & 3,95 & 0,101 & 0,0103 & 75,95 \\
\hline 6 & & & 4,1 & 0,098 & 0,0095 & 73,17 \\
\hline 7 & & & 4,2 & 0,095 & 0,0091 & 71,43 \\
\hline 8 & & & 4,5 & 0,089 & 0,0079 & 66,67 \\
\hline 9 & & & 4 & 0,100 & 0,0100 & 75,00 \\
\hline 10 & & & 4,1 & 0,098 & 0,0095 & 73,17 \\
\hline
\end{tabular}

Table 3. Constrained the deposition of the "cloud" of particles.

\begin{tabular}{|c|c|c|c|c|c|c|c|c|c|c|}
\hline $\mathrm{No}$ & $\mathrm{d}$ & $\mathrm{h}$ & $\mathrm{t}_{1}, \mathrm{~s}$ & $\mathrm{t}_{2}, \mathrm{~s}$ & $\mathrm{t}_{3}, \mathrm{~s}$ & $\mathrm{t}_{\mathrm{av}}, \mathrm{s}$ & $\mathrm{v}$ & $\mathrm{v}, \mathrm{m} / \mathrm{s}$ & $v^{*}{ }_{2}, \mathrm{~m} / \mathrm{s}$ & $\mathrm{Re}$ \\
\hline 1 & & & 4,00 & 7,5 & 20,0 & 10,5 & & 0,038 & 0,00145 & 28,57 \\
\hline 2 & & & 3,8 & 5,5 & 15,0 & 8,1 & & 0,049 & 0,00244 & 37,04 \\
\hline 3 & & & 3,8 & 5,3 & 15,0 & 8,0 & & 6,050 & 0,00248 & 37,34 \\
\hline 4 & & & 3,5 & 5,0 & 24,0 & 10,8 & & 0,037 & 0,00136 & 27,69 \\
\hline 5 & & & 4,0 & 6,2 & 12,0 & 7,4 & & 0,054 & 0,00292 & 40,54 \\
\hline 6 & & & 4,2 & 5,4 & 22,0 & 10,5 & & 0,038 & 0,00144 & 28,48 \\
\hline 7 & & & 4,1 & 4,9 & 26,0 & 11,7 & & 0,034 & 0,00118 & 25,71 \\
\hline 8 & & & 4,2 & 5,6 & 20,0 & 9,9 & & 0,040 & 0,00162 & 30,20 \\
\hline 9 & & & 3,7 & 4,9 & 15,0 & 7,9 & & 0,051 & 0,00259 & 38,14 \\
\hline 10 & 0,00075 & 0,4 & 4,0 & 5,1 & 20,0 & 9,7 & 0,000001 & 0,041 & 0,00170 & 30,93 \\
\hline
\end{tabular}

The average deposition rate of particles in the cloud was $0.043 \mathrm{~m} / \mathrm{s}$, while the deposition rate of single particles was $0.097 \mathrm{~m} / \mathrm{s}$. Thus, the rate of constrained deposition turned out to be $2 \div 2.5$ times lower than the rate of deposition of single particles. It should be emphasized that the deposition rate of single particles and the deposition rate of the first particles in the cloud almost coincide, which allows concluding that the experiment is sufficiently accurate. 


\section{Results of researches}

The results obtained for a significant difference in the speed of the constrained and free sedimentation of particles, exceeding the previously made analytical estimates, are confirmed by additional studies of the speed of the constrained sedimentation of fine suspensions at various concentrations varying more than five times.

They convincingly indicate that the sedimentation rate of suspensions at a higher initial concentration is significantly lower than the rate of sedimentation of a suspension at a lower initial concentration.

Data Presented in Fig. 4 show that during sufficiently large time intervals (from 3 to 6 hours) linear growth of the clarified layer in time takes place, which means that the suspension sedimentation rate remains constant during the experience.

This can be explained by the fact that as the upper layers were clarified, the concentration of suspended matter in the lower layer remained almost constant due to precipitation of particles from the lower layer to the bottom of the measuring vessel. The experimentally established dependence of the rate of constrained sedimentation of a fine suspension on the initial concentration is presented in Fig. 5.

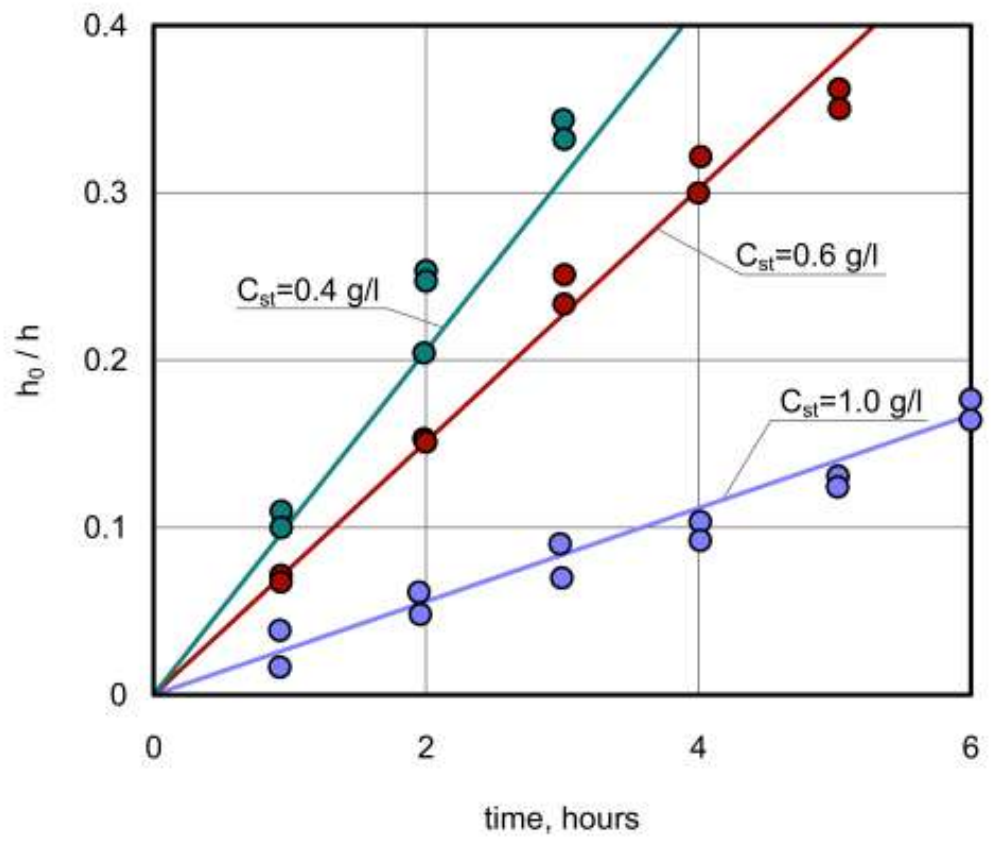

Fig. 4. Hindered settling fine slurry: h0 - a clarified layer thickness; $h$ is the total thickness of the sample layer. 


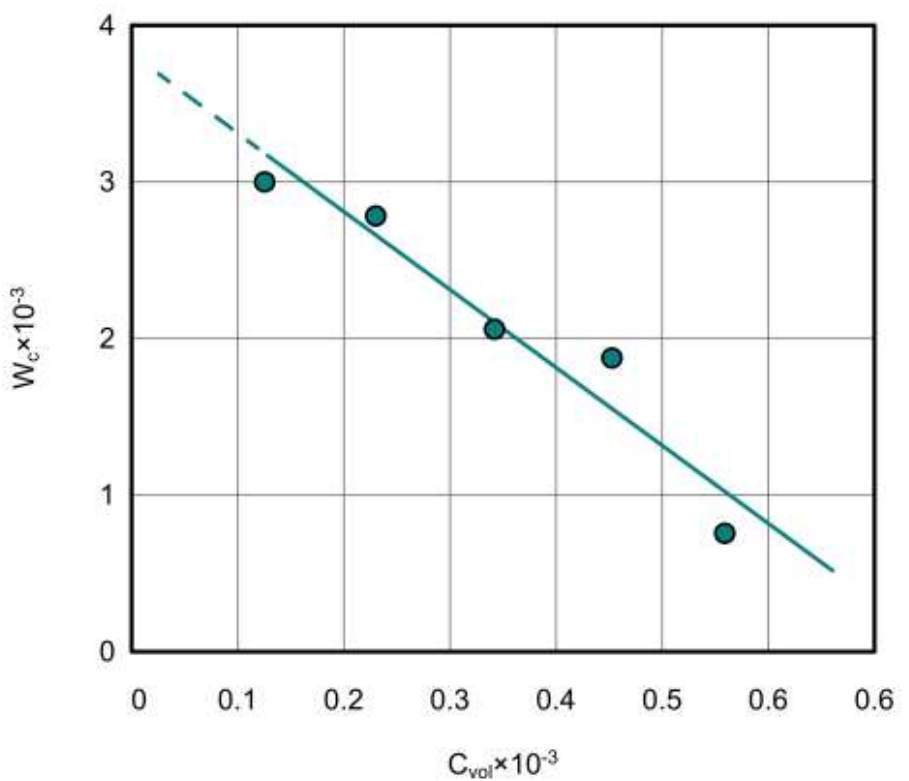

Fig. 5. The effect of concentration on the sedimentation rate of fine suspension.

\section{Conclusion}

The analysis of the obtained data allowed us to obtain the following approximation dependence for the speed of the constrained sedimentation of a fine suspension:

$$
W \frac{W_{C S}}{W_{0}}=1-1200 C_{v o l}
$$

where $C_{v o l}$ is the volume concentration of the suspension; $W_{0}$ is the rate of free sedimentation of particles.

Considerable variation of this dependence with theoretical evaluation by M. A. Velikanov made for large particles can be explained by the influence of electrodynamics' effects, which can occur between particles of the fine particulate matter in the aquatic environment, as indicated by B. V. Deryagin, W. J. London. Walker [1,6,8].

Thus, on the basis of the experimental studies carried out, a formula has been obtained for calculating the speed of the constrained sedimentation of particles of a fine suspension. It is shown that the results of experiments differ from the previously obtained theoretical estimates for a large suspension.

\section{References}

1. M.A. Volynov, V.S. Borovkov, I.M. Markova, N.V. Suykova, Vestnik MGSU 64 (2012)

2. M.A. Volynov, V.S. Borovkov, I.M. Markova V.A. Kurochkina, J.S.P of J. of S.P. of grad. and doct.st. 91 (2012)

3. M.A. Volynov, Yu.V. Bryanskaya, Trudy int. Scientific Conf. 222 (2011)

4. A.B. Klaven, Z.D. Kopaliani, Experimental research and hydraulic modeling of river flows and channel processes (Nestor History, St. Petersburg, 2011) 
5. M.M. Abou-Seida, G.H. Elsaeed, T.M.Mostafa, E.F. Elzahry, Journ. of Hydr. Res. 171 (2012)

6. I.M. Markova, Trudy int. Scientific Conf. 297 (2010)

7. Yu.V. Bryanskaya, T.G. Bogomolova, I.M. Markova, Sc. J. Academia, Architecture and construction 538 (2010)

8. I.M. Markova, Dis. deg. of cand. of tech.sc. 146 (2005)

9. S. Balasubramanian, S.L.Voropayev, H.J.S. Fernando, Journ.of Turb. 19 (2008)

10. J. Bec, L. Biferale, A. Scagliarini, A.S. Lanotte, F. Toschi, Journ. Fluid Mech. 497 (2010)

11. M. Cencini, J. Bec, L.Biferale, G.Boffetta , A. Celani, S. Musacchio, A.S. Lanotte, F. Toschi 16 (2006)

12. A. Daouadji, H. Al Gali, F. Darve, A. Zeghloul, Journ. Eng. Mech-ASCE 575 (2010)

13. J.M.N.T. Gray, B.P. Journ, Fluid Mech. 105 (2010) 